\title{
Semi Supervised Multi Kernel (SeSMiK) Graph Embedding: Identifying Aggressive Prostate Cancer via Magnetic Resonance Imaging and Spectroscopy
}

\author{
Pallavi Tiwari ${ }^{1, \star}$, John Kurhanewicz ${ }^{2}$, Mark Rosen ${ }^{3}$, and Anant Madabhushi ${ }^{1}$ \\ ${ }^{1}$ Department of Biomedical Engineering, Rutgers University, USA \\ pallavit@eden.rutgers.edu, anantm@rci.rutgers.edu \\ 2 Department of Radiology, University of California, San Francisco, USA \\ 3 Department of Radiology, University of Pennsylvania, Philadelphia, USA
}

\begin{abstract}
With the wide array of multi scale, multi-modal data now available for disease characterization, the major challenge in integrated disease diagnostics is to able to represent the different data streams in a common framework while overcoming differences in scale and dimensionality. This common knowledge representation framework is an important pre-requisite to develop integrated meta-classifiers for disease classification. In this paper, we present a unified data fusion framework, Semi Supervised Multi Kernel Graph Embedding (SeSMiKGE). Our method allows for representation of individual data modalities via a combined multi-kernel framework followed by semi- supervised dimensionality reduction, where partial label information is incorporated to embed high dimensional data in a reduced space. In this work we evaluate SeSMiK-GE for distinguishing (a) benign from cancerous $(\mathrm{CaP})$ areas, and (b) aggressive high-grade prostate cancer from indolent low-grade by integrating information from 1.5 Tesla in vivo Magnetic Resonance Imaging (anatomic) and Spectroscopy (metabolic). Comparing SeSMiK-GE with unimodal T2w, MRS classifiers and a previous published non-linear dimensionality reduction driven combination scheme (ScEPTre) yielded classification accuracies of (a) 91.3\% (SeSMiK), 66.1\% (MRI), 82.6\% (MRS) and $86.8 \%$ (ScEPTre) for distinguishing benign from $\mathrm{CaP}$ regions, and (b) 87.5\% (SeSMiK), 79.8\% (MRI), 83.7\% (MRS) and 83.9\% (ScEPTre) for distinguishing high and low grade $\mathrm{CaP}$ over a total of 19 multi-modal MRI patient studies.
\end{abstract}

\section{Introduction}

With the rapid growth of new imaging modalities and availability of multi-scale, multimodal information, data fusion has become extremely important for improved disease diagnostics. However one of the major challenges in integrating independent channels of heterogeneous information is representing them in a unified framework prior to data integration [1]. Typically, information fusing algorithms may be categorized as being either combination of data (COD) or interpretation (COI) methodologies [2]. In

\footnotetext{
* This work was supported by the Wallace H. Coulter Foundation, the National Cancer Institute under Grants R01CA136535, R21CA127186, R03CA128081, and R03CA143991, the Cancer Institute of New Jersey, Department of Defense (W81XWH-09), and Bioimagene Inc.
} 
COD, features $\mathbf{F}_{m_{1}}$ and $\mathbf{F}_{m_{2}}$ from two disparate modalities $m_{1}$ and $m_{2}$ may be combined as $\mathbf{F}_{m_{1} m_{2}}=\left[\mathbf{F}_{m_{1}}, \mathbf{F}_{m_{2}}\right]$. However directly aggregating data from very different sources without accounting for differences in the number of features and relative scaling can lead to classifier bias towards the modality with more attributes. In [3], Lanckriet et al transformed data from amino acid sequences, protein complex data, gene expression data, and protein interactions into a common kernel space. Kernels are positive definite functions which capture the similarities of the input data into a dot product space such that $K\left(\mathbf{F}\left(c_{i}\right), \mathbf{F}\left(c_{j}\right)\right)=\left\langle\Phi_{K}\left(\mathbf{F}\left(c_{i}\right), \Phi_{K} \mathbf{F}\left(c_{j}\right)\right)\right\rangle$, where $\Phi$ is the implicit pairwise embedding between points $\mathbf{F}\left(c_{i}\right)$ and $\mathbf{F}\left(c_{j}\right)$. This multi-kernel learning (MKL) (Figure 1(a)) involves similarity matrices for kernels from individual modalities being combined and used to train classifiers (within the fused kernel space) in order to make meta-predictions. However, due to the large amount of information present in each input source, all COD methods, including MKL, suffer from the curse of dimensionality.

In [1], we introduced ScEPTre (Figure 1(b)) which employed graph embedding (GE) [4] to combine low dimensional data representations obtained from individual modalities. GE accounts for the non-linearities in the data by constructing a similarity graph $G=(V, W)$, where $V$ corresponds to the vertex between pairwise points and $W$ is a $n \times n$ weight matrix of $n$ data points. However GE, like most other dimensionality reduction (DR) schemes, is unsupervised and does not include any domain knowledge while transforming the data to lower dimensions which often leads to overlapping embeddings. A few supervised DR schemes such as linear discriminant analysis (LDA) employ class label information to obtain low dimensional embeddings. However obtaining labels for biomedical data is extremely expensive and time consuming. Recently semi-supervised DR (SSDR) schemes based on GE have been proposed [5], which construct a weight matrix leveraging the known labels such that higher weights are given to within-class points and lower weights to points from different classes. The proximity of labeled and unlabeled data is then used to construct the low dimensional manifold.

In this work, we present a unified data fusion DR framework called Semi Supervised Multi Kernel Graph Embedding (SeSMiK-GE), a novel data fusion and dimensionality reduction scheme that leverages the strengths of GE, semi-supervised learning, and MKL into a single integrated framework for simultaneous data reduction, fusion, and classification. Only the work of Lin et al [6], that we are aware of, has used MKL in conjunction with GE. However their approach does not leverage learning in constructing the embeddings. SeSMiK-GE involves first transforming each individual modality in a common kernel framework, followed by weighted combination of individual kernels as $\hat{K}=\sum_{m=1}^{M} \beta_{m} K_{m}$, where $K_{m}, m \in\{1,2, \ldots, M\}$ is the kernel obtained from each modality, $\beta_{m}$ is the weight assigned to each kernel, and $M$ is the total number of kernels employed. DR is then performed on $\hat{K}$ using semi-supervised GE (SSGE) which incorporates partial labels to provide a better low dimensional representation of the data allowing for better class separation and hence improved classification with limited training samples.

In this paper we show an application of SeSMiK-GE to combine structural information obtained from T2-weighted Magnetic Resonance (MR) Imaging (T2w MRI) and metabolic information obtained from MR Spectroscopy (MRS) for detection of highgrade prostate cancer $(\mathrm{CaP})$ in vivo. The Gleason grading system is the most commonly used system world-wide for identifying aggressivity of $\mathrm{CaP}$, and hence patient outcome. 


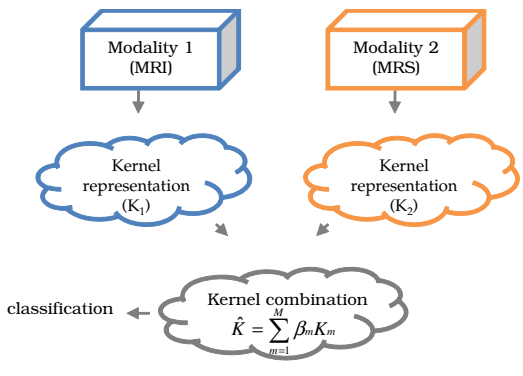

(a) Multi-kernel learning

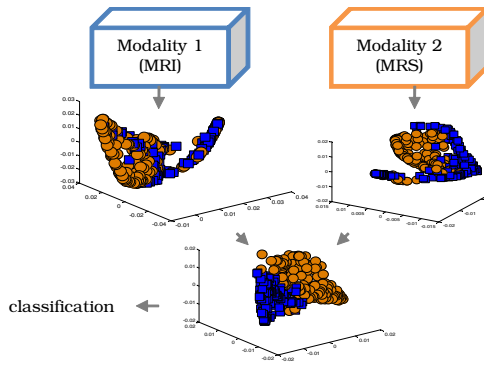

(b) ScEPTre

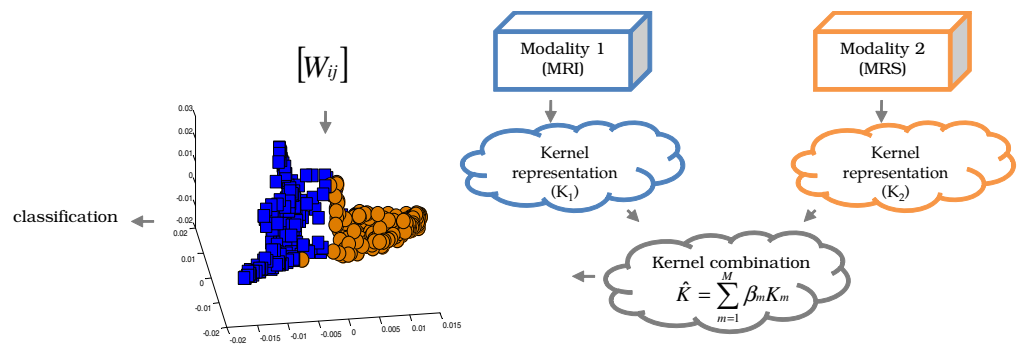

Fig. 1. (a) MKL employs a kernel combination strategy, (b) ScEPTre-based data fusion where low dimensional embedding representations are combined, and (c) SeSMiK-GE method where MKL is performed to first combine the data in a common kernel space followed by semi-supervised GE. The two colors in each 3D embedding plot represent two different classes.

High Gleason scores are associated with poor outcome, while lower scores are typically associated with better patient outcome. Recently, researchers have been attempting to identify MR imaging signatures for high- and low-grade $\mathrm{CaP}$ in vivo [7/8].

\section{Graph Embedding Framework}

The aim of GE [4] is to reduce the data matrix $\mathscr{F} \in \mathbb{R}^{D}$ into a low-dimensional space $\mathbf{y} \in \mathbb{R}^{d}(D>>d)$, such that object adjacencies are preserved from $\mathbb{R}^{D}$ to $\mathbb{R}^{d}$. Let $\mathscr{F}=\left[\mathbf{F}\left(c_{1}\right), \mathbf{F}\left(c_{2}\right), \ldots, \mathbf{F}\left(c_{n}\right)\right] \in \mathbb{R}^{D}$ be a data matrix of $n$ objects, $i \in\{1, \ldots, n\}$, with dimensionality $D$, and $\mathbf{y}=\left[y_{1}, y_{2}, \ldots, y_{n}\right]$ be the corresponding optimal low dimensional projection matrix. $\mathbf{y}$ can be obtained by solving,

$$
\mathbf{y}=\arg \min _{y}\left(\sum_{i, j=1}^{n}\left\|y_{i}-y_{j}\right\|^{2} w_{i j}\right),
$$

where $W=\left[w_{i j}\right]$ is a similarity matrix which assigns edge weights to characterize similarities between pairwise points $c_{i}$ and $c_{j}, i, j \in\{1, \ldots, n\}$. The minimization of Equation 1 reduces it to an eigenvalue decomposition problem,

$$
W \mathbf{y}=\lambda \mathscr{D} \mathbf{y}
$$


where $\mathscr{D}$ is a diagonal matrix, $\mathscr{D}_{i i}=\sum_{i} W_{i j}$. According to the Representer Theorem [9], to calculate the kernel representation $K\left(\mathbf{F}\left(c_{i}\right), \mathbf{F}\left(c_{j}\right)\right)$ of input data, it is assumed that the optimal embedding $\mathbf{y}$ lies in the input space such that $\mathbf{y}=\sum_{j=1}^{n} \alpha_{j} K\left(\mathbf{F}\left(c_{i}\right), \mathbf{F}\left(c_{j}\right)\right)$. Thus, the kernel formulation of Equation 2 can be re-written as,

$$
K W K^{T} \alpha=\lambda K \mathscr{D} K^{T} \alpha,
$$

where $K$ is a valid positive semi-definite kernel and $\alpha$ is the $d$ dimensional eigenvector of the kernel representation in Equation 3.

\section{Semi-Supervised Multi-kernel Graph Embedding (SeSMiK-GE)}

1. Constructing Kernels for each modality: Kernel functions embed input data in the implicit dot product space, evaluating which yields a symmetric, positive definite matrix (gram matrix). A kernel gram matrix $K_{m}$ defining the similarities between $n$ data points in each modality $m$ may be obtained as $K_{m}=\left[K\left(\mathbf{F}\left(c_{i}\right), \mathbf{F}\left(c_{j}\right)\right)\right] \forall i, j \in\{1, \ldots, n\}$.

2. Combining Multiple kernels: A linear combination of different kernels has the advantage of also yielding a symmetric, positive definite matrix. Assuming we have $M$ base kernel functions for $M$ modalities, $\left\{K_{m}\right\}_{m=1}^{M}$, corresponding individual kernel weights $\beta_{m}$, the combined kernel function may be expressed as,

$$
\begin{aligned}
\hat{K}\left(\mathbf{F}\left(c_{i}\right), \mathbf{F}\left(c_{j}\right)\right) & =\sum_{m=1}^{M} \beta_{m} K_{m}\left(\mathbf{F}\left(c_{i}\right), \mathbf{F}\left(c_{j}\right)\right), \beta_{m} \geq 0, \forall(i, j) \in\{1, \ldots, n\} \\
& =\sum_{m=1}^{M} \beta_{m} K_{m}, \beta_{m} \geq 0 .
\end{aligned}
$$

3. Constructing the adjacency graph using partial labels: Assuming the first $l$ of $n$ samples are labeled $\omega_{l} \in\{+1,-1\}$, we can incorporate the partial known labels into the similarity matrix $W=\left[w_{i j}\right]$. A $\mathscr{N}$ nearest neighbor graph, $\mathscr{N}>0$, is created to obtain $W$ such that pairwise points in $\mathscr{N}$ neighborhood with same labels are given high weights and points with different class labels are given low weights [5]. If the points are not in $\mathscr{N}$, the corresponding edges are not connected. Thus the weight matrix is,

$$
w_{i j}= \begin{cases}\gamma(1+\gamma), & \text { if } F\left(c_{i}\right) \in \mathscr{N}_{j} \text { or } F\left(c_{j}\right) \in \mathscr{N}_{i} \text { and } \omega_{i}=\omega_{j}, \\ \gamma(1-\gamma), & \text { if } F\left(c_{i}\right) \in \mathscr{N}_{j} \text { or } F\left(c_{j}\right) \in \mathscr{N}_{i} \text { and } \omega_{i} \neq \omega_{j}, \\ \gamma, & \text { if } F\left(c_{i}\right) \in \mathscr{N}_{j} \text { or } F\left(c_{j}\right) \in \mathscr{N}_{i}, i>l \text { or } j>l, \\ 0, & \text { otherwise. }\end{cases}
$$

where $\gamma=e^{\frac{\left\|F(c) i_{i}-F(c) j\right\|^{2}}{\sigma}}$ and $\sigma$ is the scaling parameter. The weight matrix $W_{m}$ obtained from each modality may be averaged to obtain $\hat{W}=\frac{1}{m} \sum_{m=1}^{M} W_{m}$.

4. Obtaining the low dimensional embedding: The combined kernel $\hat{K}$ and associated weight matrix $\hat{W}$ obtained from Equations (4) and (5) can be used to reduce Equation (3) to the eigenvalue decomposition problem,

$$
\hat{K} \hat{W} \hat{K}^{T} \alpha=\lambda \hat{K} \tilde{\mathscr{D}} \hat{K}^{T} \alpha,
$$

where $\tilde{\mathscr{D}}=\sum_{j} \hat{w}_{j i}$. The optimal $d$ dimensional eigenvectors $\alpha=\left\{\alpha_{1}, \alpha_{2}, \ldots, \alpha_{n}\right\}$ are obtained using standard kernel ridge regression optimization as described in [10]. 


\section{SeSMiK-GE for Prostate Cancer Detection and Grading}

\subsection{Data Description}

A total of 191.5 Tesla (T) T2w MRI and corresponding MRS pre-operative endorectal in vivo prostate studies were obtained from the University of California, San Francisco. The 3D prostate T2w MRI scene is represented by $\hat{\mathscr{C}}=(\hat{C}, \hat{f})$, where $\hat{C}$ is a $3 \mathrm{D}$ grid of voxels $\hat{c} \in \hat{C}$ and $\hat{f}(\hat{c})$ is a function that assigns an intensity value to every $\hat{c} \in \hat{C}$. We also define a spectral scene $\mathscr{C}=(\boldsymbol{C}, \boldsymbol{F})$ where $C$ is a 3D grid of MRS metavoxels, $c \in C$, and $\boldsymbol{F}$ is a spectral vector associated with each $c \in C$. Note that multiple MRI resolution voxels are present within the region $R_{c d}$ between any two adjacent MRS resolution metavoxels $c, d \in C$. An expert spectroscopist (JK) manually annotated individual MRS metavoxels across all 19 patient studies as firstly (a) CaP/benign, and secondly, (b) as low/high grade $\mathrm{CaP}$. The $191.5 \mathrm{~T}$ studies comprised a total of (a) $573 \mathrm{CaP}$ and 696 benign metavoxels, and (b) 175 low and 96 high grade CaP metavoxels.

\subsection{Feature Extraction from MRI and MRS}

(a) Feature extraction from MRS: For each $c \in C, \boldsymbol{F}(c)=\left[f_{a}(c) \mid a \in\{1, \ldots U\}\right]$, represents the MR spectral vector, reflecting the frequency component of each of $U$ metabolites. The corresponding spectral data matrix is given as $\mathscr{F}=\left[\mathbf{F}_{1}(c) ; \mathbf{F}_{2}(c), \ldots ; \mathbf{F}_{n}(c)\right]$ $\in \mathbb{R}^{n \times U}$ where $n=|C|,|C|$ is the cardinality of $C$.

(b) Feature extraction from MRI: 38 texture features were extracted to define CaP appearance on in vivo T2w MRI [11]. We calculated the feature scenes $\hat{\mathscr{G}}_{u}=\left(\hat{C}, \hat{f}_{u}\right)$ for each $\hat{\mathscr{C}}$ by applying the feature operators $\Phi_{u}, u \in\{1, \ldots, 38\}$ within a local neighborhood associated with every $\hat{c} \in \hat{C}$. 13 gradient, 12 first order statistical and 13 Haralick features were extracted at each $\hat{c} \in \hat{C}$. We define a T2w MRI texture feature vector for each metavoxel $c \in C$ by taking the average of the feature values within the corresponding metavoxel as $g_{u}(c)=\frac{1}{\left|R_{c d}\right|} \sum_{\hat{c} \in R_{c d}}\left[\hat{f}_{u}(\hat{c})\right]$. The corresponding feature vector is then given as $\boldsymbol{G}(c)=\left[g_{u}(c) \mid u \in\{1, \ldots, 38\}\right], \forall c \in C$, and the MRI data matrix is given as $\mathscr{G}=\left[\boldsymbol{G}_{1} ; \boldsymbol{G}_{2} ; \ldots ; \boldsymbol{G}_{n}\right] \in \mathbb{R}^{n \times 38}$.

\subsection{SeSMiK-GE for Integration of MRI and MRS}

A Gaussian kernel $K\left(\mathbf{F}\left(c_{i}\right), \mathbf{F}\left(c_{j}\right)\right)=e^{\frac{\left\|\mathbf{F}\left(c_{i}\right)-\mathbf{F}\left(c_{j}\right)\right\|^{2}}{\sigma}}$ was employed within SeSMiK-GE to obtain $K_{M R S}$ and $K_{M R I}$ from input MRS data $\mathscr{F}$ and MRI data $\mathscr{G} .40 \%$ of the total samples were randomly selected to train the algorithm over 25 iterations of cross validation. $W_{M R I}$ and $W_{M R S}$ were obtained using Equation (5) and averaged to obtained the fused gram matrix $\hat{W}$. The algorithm was evaluated over different values of $\beta \in[0,1]$ over intervals of 0.1 to obtain 11 embedding outputs $\alpha_{q}, q \in\{1, \ldots, 11\}$, where $\alpha_{1}$ represents the embedding obtained purely from $K_{M R S}(\beta=0)$ and $\alpha_{11}$ represents the embedding obtained purely from $K_{M R I}(\beta=1)$. A probabilistic boosting tree (PBT) classifier [12], was then trained using the same set of samples exposed for SeSMiK over each iteration of 25 cross validation runs. During each iteration, the optimal $\alpha_{q}$ which results in the maximum classification accuracy is selected as the final embedding result. The algorithm for SeSMiK-GE is presented below. 
Algorithm SeSMiK-GE

Input: $\mathscr{F}, \mathscr{G}, \mathscr{N}, d$

Output: $\alpha$

begin

0. Obtain $K_{M R S} \leftarrow \mathscr{F}$ and $K_{M R I} \leftarrow \mathscr{G}$

1. Obtain $W_{M R I}$ and $W_{M R S}$ using $\mathscr{N}$; obtain $\hat{W}$ using Equation (6)

2. Initialize $\beta=0, q=1$

3. while $\beta=1$, compute $\hat{K}_{q}=\beta \times K_{M R I}+(1-\beta) \times K_{M R S}$

4. Substitute $\hat{K}_{q}$ and $\hat{W}$ in Equation (3)

5. Obtain $d$-dimensional $\alpha_{q}$ by solving Equation (7)

6. return $\alpha_{q}$

7. $\beta=\beta+0.1, q=q+1$

8. endwhile

end

The algorithm above was applied to the problems of (a) discriminating $\mathrm{CaP}$ vs. benign regions, and (b) identifying high-grade $\mathrm{CaP}$ using multi-protocol MRI.

\section{Results and Discussion}

\subsection{Qualitative}

A PBT classifier [12] was trained on the low dimensional embedding representations obtained from application of SeSMiK-GE to the 19 MRI, MRS studies. Figure 2(a) shows a T2w MRI slice with high grade CaP, while Figure 2(b) shows signature spectra corresponding to low and high grade $\mathrm{CaP}$, in turn illustrating the difficulty in visually

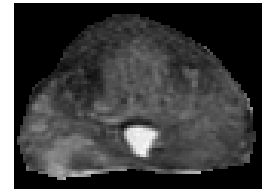

(a)



(e)

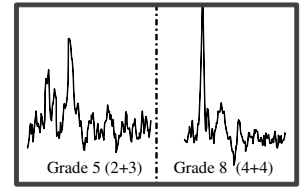

(b)



(f)



(c)

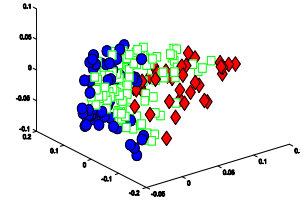

(g)



(d)

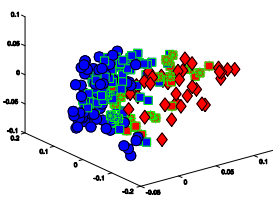

(h)

Fig. 2. (a) Original T2w MRI, (b) MRS signatures for high/low grade CaP. Red regions on (c)-(d) show the classification results obtained using SeSMiK-GE for identifying CaP and high grade $\mathrm{CaP}$ on in vivo MRI, MRS. White ROI delineates the ground truth for CaP extent in (c) and highgrade $\mathrm{CaP}$ in (d). (e), (g) show 3D embedding plots obtained from SeSMiK-GE (with partial training labels for each class) for cancer metavoxels (red) and benign metavoxels (blue). The spectra in the evaluation (test set) are shown via green squares. (f), (h) illustrate the classification results via PBTs on the same embedding for detection of $\mathrm{CaP}((\mathrm{f}))$, and high-grade $\mathrm{CaP}((\mathrm{h}))$. 
identifying high grade $\mathrm{CaP}$ on in vivo MRI and MRS. Figures 2(c)-(d) show the PBT classifier prediction results (as red regions) on the same T2w MRI slice using SeSMiKGE for identifying CaP (Figure 2(c)) and high-grade CaP (Figure 2(d)). Note the high detection accuracy obtained using SeSMiK-GE for both CaP (Figure 2(c)) and highgrade $\mathrm{CaP}$ (Figure 2(d)) identification. Ground truth for $\mathrm{CaP}$ and high-grade $\mathrm{CaP}$ extent is shown via a white ROI on Figures 2(c) and 2(d).

Embedding plots obtained from SeSMiK-GE for cancer (red)/benign (blue) and high (red)/low (blue) grade CaP are shown in Figures 2(e)-(h). Figures 2 (e), (g) show the partial labels provided to SeSMiK-GE, allowing for better separation between the classes (green squares represent the unlabeled samples). Figures 2(f), (h) show corresponding PBT classification labels for identifying $\mathrm{CaP}(2(\mathrm{f}))$ and high-grade $\mathrm{CaP}(2(\mathrm{~h}))$. Note that in Figures 2(f) and (h), a majority of the unlabeled samples are accurately identified by our scheme, despite using limited partial labels for training.

\subsection{Quantitative}

Table 1(a) shows mean area under the ROC curve (AUC) and accuracy results averaged over 19 studies for identifying cancer vs. benign using SeSMiK-GE on (a) only MRI, (b) only MRS, and (c) in combining MRS and MRI (results shown are for optimal $\alpha_{q}$ obtained at $\beta=0.8$ ). We compared our results with ScEPTre [1], where GE [4] was first performed on each of $\mathscr{F}$ and $\mathscr{G}$ followed by concatenation of the resulting low

Table 1. (a) Average AUC and accuracy for CaP detection, compared to MRI-MRS alone, and ScEPTre [1] based data fusion, averaged over a total of 19 MRI-MRS studies using the 30 topranked eigen values, (b) Average CaP detection accuracy and AUC results of SeSMiK-GE and ScEPTre for different dimensions $\mathbf{d} \in\{10,20,30\}$.

(a)

\begin{tabular}{|c|c|c|}
\hline Method & AUC & Accuracy \\
\hline T2w MRI & $66.1 \pm 1.5$ & $61.9 \pm 1.3$ \\
\hline MRS & $82.6 \pm 1.3$ & $76.8 \pm 1.3$ \\
\hline ScEPTre & $86.8 \pm 1.26$ & $78.2 \pm 1.2$ \\
\hline SeSMiK-GE & $\mathbf{9 1 . 3} \pm \mathbf{0 . 2}$ & $\mathbf{8 3 . 0} \pm \mathbf{0 . 1}$ \\
\hline
\end{tabular}

(b)

\begin{tabular}{|c|c|c|c|c|}
\hline d & \multicolumn{2}{|c|}{ AUC } & \multicolumn{2}{c|}{ Accuracy } \\
\hline & SeSMiK & ScEPTre & SeSMiK & ScEPTre \\
\hline 10 & $89.8 \pm 0.8$ & $86.8 \pm 0.9$ & $84.2 \pm 1.1$ & $80.6 \pm 1.3$ \\
\hline 20 & $90.7 \pm 0.9$ & $87.5 \pm 0.8$ & $84.6 \pm 0.1$ & $79.1 \pm 1.2$ \\
\hline 30 & $\mathbf{9 1 . 3} \pm \mathbf{0 . 2}$ & $86.8 \pm 1.26$ & $\mathbf{8 3 . 0} \pm \mathbf{0 . 1}$ & $78.2 \pm 1.2$ \\
\hline
\end{tabular}

Table 2. (a) Average AUC and accuracy for high-grade CaP detection, compared to MRI or MRS alone, and ScEPTre [1] data fusion averaged over a total of 19 MRI-MRS studies using the 10 top-ranked eigen values, (b) high-grade CaP detection accuracy and AUC results of SeSMiK-GE and ScEPTre for different dimensions $\mathbf{d} \in\{10,20,30\}$.

(a)

\begin{tabular}{|c|c|c|}
\hline Method & AUC & Accuracy \\
\hline T2w MRI & $79.8 \pm 3.3$ & $74.1 \pm 4.0$ \\
\hline MRS & $83.7 \pm 3.5$ & $78.5 \pm 3.0$ \\
\hline ScEPTre & $83.9 \pm 3.5$ & $76.8 \pm 3.1$ \\
\hline SeSMiK-GE & $\mathbf{8 7 . 5} \pm \mathbf{2 . 5}$ & $\mathbf{8 2 . 5} \pm \mathbf{2 . 6}$ \\
\hline
\end{tabular}

(b)

\begin{tabular}{|c|c|c|c|c|}
\hline $\mathbf{d}$ & \multicolumn{2}{|c|}{ AUC } & \multicolumn{2}{c|}{ Accuracy } \\
\hline & SeSMiK & ScEPTre & SeSMiK & ScEPTre \\
\hline 10 & $86.9 \pm 2.2$ & $84.4 \pm 2.7$ & $80.5 \pm 2.6$ & $79.1 \pm 3.6$ \\
\hline 20 & $\mathbf{8 7 . 5} \pm \mathbf{2 . 5}$ & $83.9 \pm 3.5$ & $\mathbf{8 2 . 5} \pm \mathbf{2 . 6}$ & $76.8 \pm 3.1$ \\
\hline 30 & $86.5 \pm 2.8$ & $83.8 \pm 3.5$ & $79.5 \pm 3.3$ & $77.2 \pm 3.5$ \\
\hline
\end{tabular}


dimensional eigenvectors. Note the high detection accuracy obtained using SeSMiKGE for $\mathrm{CaP}$ (Table 1(a)) and high-grade $\mathrm{CaP}$ (Table 2(a)) detection. Table 1(b) shows the low variability in AUC and accuracy results over different values of reduced dimensions (d). Similar results for discriminating high and low grade $\mathrm{CaP}$ are shown in Table 2(b).

\section{Conclusions and Future Work}

We presented a novel semi-supervised multi-kernel (SeSMiK) scheme which is well integrated in a graph embedding framework for simultaneous data fusion and dimensionality reduction. Multi-kernel learning is first used to combine heterogeneous information from various data sources in a common kernel framework. The method leverages partial domain knowledge to create an optimal embedding from the combined data such that object classes are optimally separable. We demonstrated the application of our scheme in discriminating cancer/benign and high/low grade prostate cancer regions using metabolic information obtained from MRS and anatomic information obtained from T2w MRI. Quantitative results demonstrate a high detection accuracy in identifying cancer and high-grade prostate cancer regions, suggesting that SeSMiK can serve as a powerful tool for both computer aided diagnosis and prognosis applications. In future work we intend to explore the application of SeSMiK in other domains and problems.

\section{References}

1. Tiwari, P., et al.: Spectral Embedding Based Probabilistic Boosting Tree (ScEPTre): Classifying High Dimensional Heterogeneous Biomedical Data. In: Yang, G.-Z., Hawkes, D., Rueckert, D., Noble, A., Taylor, C. (eds.) MICCAI 2009. LNCS, vol. 5762, pp. 844-851. Springer, Heidelberg (2009)

2. Rohlfing, T., et al.: Information fusion in biomedical image analysis: Combination of data vs. combination of interpretations. In: Christensen, G.E., Sonka, M. (eds.) IPMI 2005. LNCS, vol. 3565, pp. 150-161. Springer, Heidelberg (2005)

3. Lanckriet, G., et al.: Kernel-Based Data Fusion and Its Application to Protein Function Prediction in Yeast. In: Pacific Symposium on Biocomputing, pp. 300-311 (2004)

4. Shi, J., Malik, J.: Normalized Cuts and Image Segmentation. IEEE Transactions on Pattern Analysis and Machine Intelligence 22, 888-905 (2000)

5. Zhao, H.: Combining labeled and unlabeled data with graph embedding. Neurocomputing 69(16-18), 2385-2389 (2006)

6. Lin, Y., et al.: Dimensionality Reduction for Data in Multiple Feature Representations. In: Proc. NIPS, vol. 21, pp. 961-968 (2008)

7. Zakian, K., et al.: Correlation of Proton MRSI with Gleason Score Based on Step-Section Pathologic Analysis after Radical Prostatectomy. Radiology 234(3), 804-814 (2005)

8. Wang, L., et al.: Assessment of Biologic Aggressiveness of Prostate Cancer: Correlation of MRI with Gleason Grade after Radical Prostatectomy. Radiology 246(1), 168-176 (2008)

9. Schlkopf, B., et al.: A Generalized Representer Theorem. In: Proc. Computational Learning Theory, pp. 416-426 (2001)

10. Cai, D., et al.: Semi-Supervised Discriminant Analysis. In: Proc. ICCV, pp. 1-7 (2007)

11. Madabhushi, A., et al.: Automated detection of prostatic adenocarcinoma from highresolution ex vivo mri. IEEE Transactions on Medical Imaging 24(12), 1611-1625 (2005)

12. Tu, Z.: Probabilistic Boosting-Tree: Learning Discriminative Models for Classification, Recognition, and Clustering. In: Proc. ICCV, vol. 2, pp. 1589-1596 (2005) 